\title{
An Evaluation on Fracture Toughness in SUS304 at High Strain Rate Considering Process Zone
}

\author{
Shiguma Yoshida $^{1}$ and Takeshi Iwamoto ${ }^{2 *}$ \\ ${ }^{1}$ Graduate School of Advanced Science and Engineering, Hiroshima University, Hiroshima, Japan \\ ${ }^{2}$ Academy of Science and Technology, Hiroshima University, Hiroshima, Japan
}

\begin{abstract}
Some research works report the relationships between transformation toughening and process zone. In SUS304, the reduction of transformation toughening at high strain rate is expected from the result of a small punch test. Currently, the process zone in SUS304 is fuzzily defined. Therefore, a consideration of damaging process is necessary in order to understand a fracture mechanism associated with transformation toughening and process zone. In this study, at first, tensile tests of pre-cracked sheet specimens made of SUS304 are conducted by the split Hopkinson pressure bar in order to understand the fracture mechanism phenomenologically at high deformation rate. During the test, a DC potential difference method is introduced to capture onset time of fracture.
\end{abstract}

\section{Introduction}

A mechanism of transformation toughening in ceramics is studied in the past. Sigl et al. [1] simulate the relationship between a size of process zone and stress intensity factor in WC by introducing ductile phase around crack tip. From this result, it is considered that the transformation toughening induces the reduction of stress concentration around crack tip by an increase in a shielding effect.

On the other hand, Pham and Iwamoto [2] conduct a small punch test by using tiny disk specimens made of SUS304 at various strain rate. In addition, Talonen et al. [3] report that martensitic phase is suppressed at high strain rate by conducting tensile tests targeted on a smooth specimen of SUS304 in a wide range of strain rate. Their results tell us that rate sensitivity of its fracture toughness gets negative because of heat generation. From these studies, the transformation toughening in SUS304 at high strain rate will be reduced as considered above.

The process zone in SUS304 can be fuzzily defined by a finite region with strong inhomogeneity including crack healing, microcrack propagation, transformation region, etc. Strictly speaking, an evaluation of fracture process at around crack tip is difficult due to the fuzziness. Therefore, a study about the fracture mechanism associated with the process zone is quite important because the discussion on coupling between the phenomena in inhomogeneous region at around crack tip and transformation toughening is insufficient. The

*Corresponding author: iwamoto@mec.hiroshima-u.ac.jp 
additional consideration of damaging process is necessary in order to express strong inhomogeneity on the deformation around crack tip.

In this study, to achieve the goal mentioned above, at first, tensile tests of pre-cracked sheet specimens made of SUS304 are conducted by the split Hopkinson pressure bar (SHPB) in order to understand the fracture mechanism phenomenologically at high deformation rate. During the test, a DC potential difference method is introduced to capture onset time of fracture.

\section{Experimental principle and procedure}

\subsection{Split-Hopkinson pressure bar (SHPB) method}

Direct impact tensile testing machine based on SHPB method is applied in this study. Input and output bars are made of same material. However, it is currently considered their diameter is different. The stress waves defined by $\sigma_{1}(t)$ and $\sigma_{2}(t)$ in the input and output bars are obtained as

$$
\sigma_{1}(t)=\frac{A_{1}}{A_{0}} E\left\{\varepsilon_{i}(t)+\varepsilon_{r}(t)\right\} \text { and } \sigma_{2}(t)=\frac{A_{2}}{A_{0}} E \varepsilon_{t}(t)
$$

where $A_{0}, A_{1}$ and $A_{2}$ are the cross-sectional area of the specimen, input bar and output bar, $\varepsilon_{i}(t), \varepsilon_{r}(t)$ and $\varepsilon_{t}(t)$ are input, reflection and transmitted strain waves. If the relative velocity at the ends of bars is equal to tensile rate in specimen, strain rate $\dot{\varepsilon}_{n}(t)$ can be expressed as

$$
\varepsilon_{n}(t)=\int_{0}^{t} \dot{\varepsilon}_{n}(t) d t \text { and } \dot{\varepsilon}_{n}(t)=\frac{c}{l_{0}}\left\{\varepsilon_{i}(t)-\varepsilon_{r}(t)-\varepsilon_{t}(t)\right\}
$$

Here, $\rho, c$, and $l_{0}$ are density, longitudinal elastic wave rate, and specimen length. Nominal stress in specimen $\sigma_{n}$ is the average value of Eq. (1) which can be expressed

$$
\sigma_{n}(t)=\frac{\sigma_{1}(t)+\sigma_{2}(t)}{2}=\frac{E}{2 A_{0}}\left[A_{1}\left\{\varepsilon_{i}(t)+\varepsilon_{r}(t)\right\}+A_{2} \varepsilon_{t}(t)\right]
$$

\subsection{Detecting onset time of fracture}

In order to detect the time for fracture initiation, a DC potential difference method is introduced in this study. The time history of output voltage obtained from the wire attached around the crack tip is integrated to remove the noise with high frequency. The approximation by a polynomial equation is applied to the integrated curve. The accelerating change in voltage can be captured by taking a fourth-order time derivative for the approximated curve. The time when the acceleration changes from positive to negative or vice versa can be detected at the intersections of the curve and time axis. The latest intersection is chosen as the onset time of fracture because the voltage is expected to increase monotonically after fracture.

\subsection{Experimental procedure}

The fracture toughness is evaluated by J-integral in this study. J-integral under mode I loading can be calculated by the simplified equation by Rice [4] with an assumption of the path independence and experimentally-obtained force-displacement curves. Fig. 1 shows the geometry of specimen for tensile tests. The thickness is $0.5 \mathrm{~mm}$ as same as a conventional specimen of the small punch test. The specimens are mainly fixed at fillet part and supported auxiliary by four pins with small diameter. Based on ASTM standard, a slit with $2.5 \mathrm{~mm}$ in 
width is introduced in the center of the specimen by using the electrical discharge machining. A pre-crack with additional $2.5 \mathrm{~mm}$ in total width is inserted by using of the fatigue testing machine (Shimadzu, Servopulser EHF-EV200kN). Because their microstructure may have been changing by not only machining but also the insertion process of the pre-crack, the solution heat treatment is conducted under the condition at $1323 \mathrm{~K}$ for 30 minutes followed by the immediate quenching. Fig. 2 shows the apparatus near the specimen for an impact test. $4 \mathrm{~mm}$ in the diameter of output bar is much smaller than the input bar because the thin specimens may result in the reduced transmitted wave. $147 \mathrm{~s}^{-1}$ of strain rate can be obtained by controlling the pressure in the air tank.

\section{Result and discussion}

Fig. 3 shows the time history of output voltage at $147 \mathrm{~s}^{-1}$ by the DC potential method and the fourth derivative of approximated curve. A good agreement between the time at rising the voltage and the latest intersection can be observed. This is a supporting evidence which the onset of crack progress can be detected by the DC potential method. Fig. 4 shows the relationships between nominal stress and strain under impact condition. In this figure, the curves under quasi-static loading are also plotted as a comparison. Maximum nominal strain is determined by Fig. 3. Maximum nominal stress is indicated negative rate sensitivity clearly. On the other hand, maximum nominal strain weakly shows negative rate sensitivity. However, nominal stress under impact loading is extremely lower and the sensitivity of maximum nominal strain is turned negative to positive between impact and quasi-static loading. Fig. 5 shows the relationship between J-integral and strain rate. The negative rate sensitivity of fracture toughness can be observed.

\section{Summary}

In this study, impact tensile tests of pre-cracked sheet specimens made of SUS304 were conducted by the split Hopkinson pressure bar in order to evaluate the rate sensitivity of fracture toughness at various strain rate. Negative rate sensitivity of J-integral was indicated. Other detailed results will be shown and discussed at the conference.

\section{Acknowledgement}

We gratefully acknowledge a financial support from a fund by Higashi-Hiroshima city.

\section{References}

1. L. G. Sigl, P. A. Mataga, B. J. Dalglish, R. M. Mcmeeking, A. G. Evans, Acta Metall. 36 (1988), 945-953.

2. H. T. Pham, T. Iwamoto, ISIJ Int., 55 (2015), 2661-2666.

3. J. Talonen, H. Hänninen, P. Nenonen, G. Pape, Metall. Mater. Trans. A, 36 (2005).

4. J. R. Rice, J. Appl. Mech. 35 (1968), 379-386. 


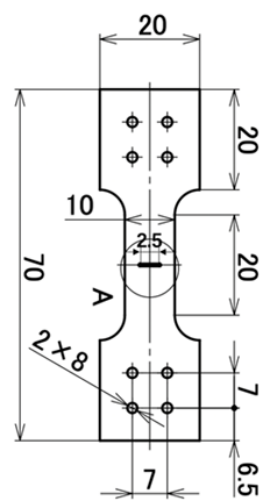

Fig. 1 Geometry of specimen for tensile test

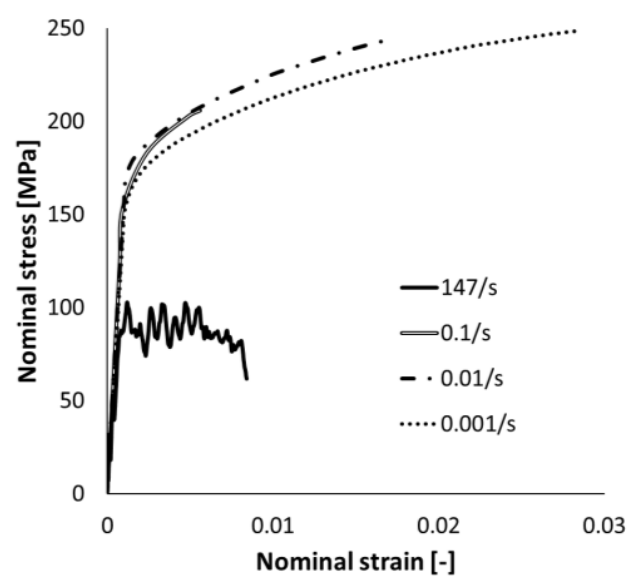

Fig. 4 Nominal stress-strain curve at various strain rate

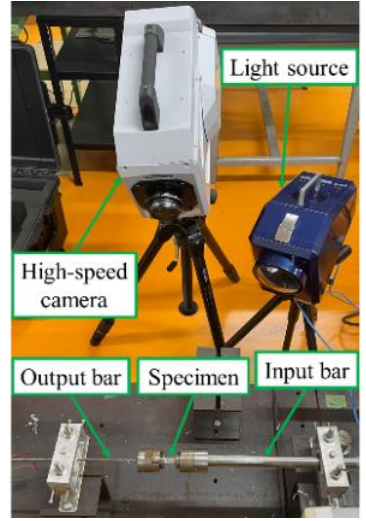

Fig. 2 Apparatus near the specimen for an impact test

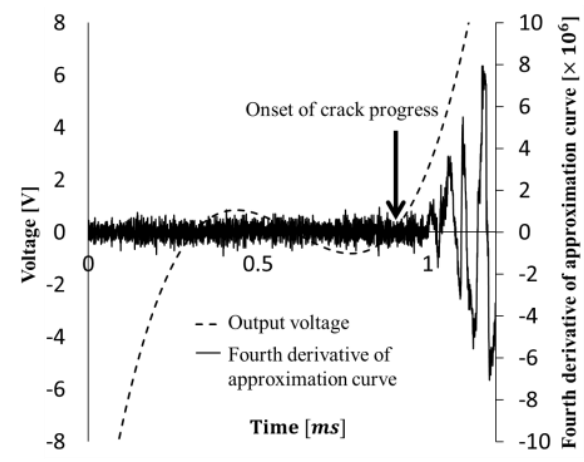

Fig. 3 Time history of output voltage at $147 \mathrm{~s}^{-1}$ by DC potential difference method and the fourth-derivative of approximated curve

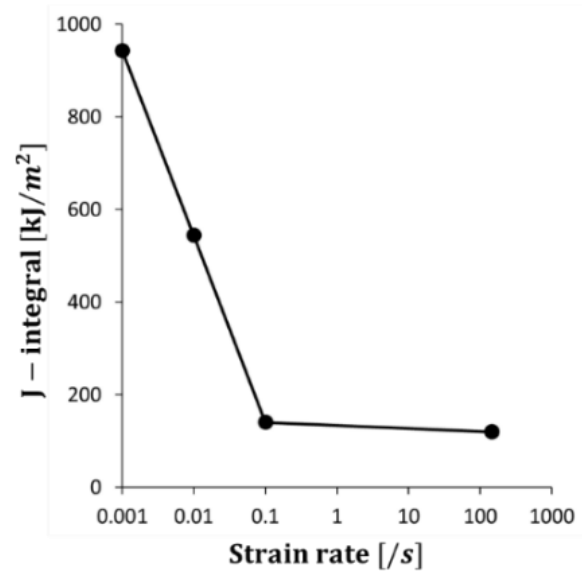

Fig. 5 Relationship between J-integral and strain rate 